\title{
Comparison between Different Electrical Configurations of Emergency Diesel Generators for Redundancy and Reliability Improving
}

\author{
Stefano Elia1*, Ezio Santini', Manuel Tobia \\ 1 Department of Astronautical, Electrical and Energy Engineering, Faculty of Civil and Industrial Engineering, Sapienza University, \\ Via delle Sette Sale 12b, 00184 Rome, Italy \\ *Corresponding author, e-mail: stefano.elia@uniroma1.it
}

Received: 02 October 2018, Accepted: 26 October 2018, Published online: 30 November 2018

\begin{abstract}
The failure of an emergency diesel generator could cause a blackout with economic or human losses, especially if it occurs in a hospital. The authors performed an up to date statistic on diesel generators fails has been investigated in a hundred of hospitals. Although standard maintenance operation has been correctly performed, it has been found a $6 \%$ of failures for the genset per year. MTBF of generators and main components have been investigated and updated values are reported in this paper. Components reliability is proved to be at its maximum technological value, consequently an innovative solution is here proposed. Often neighboring buildings or different electrical systems have separated emergency generators. Nowadays, in case of genset failure, most of the electrical installations are not designed to be fed from other generators. Several electrical configurations for diesel generators interconnections have been studied, in order to guarantee the mutual succor between them and therefore improving reliability. Two innovative schemes for emergency generators connection are here proposed for a more reliable electrical design.
\end{abstract}

Keywords

electrical power plants, diesel generators, essential electrical systems, MTBF, reliability

\begin{abstract}
1 Introduction
Innovative connections between diesel gensets have been studied. The proposed configurations allow the load to be fed by another neighboring genset in case of relevant generator's failure. This choice is due to the barely possibility to obtain genset and plant components further reliability improvement. Each generator must have a suitable rated power to support both loads. This is an essential hypothesis to install the proposed system. However, in case of small generators, an automatic cutoff for non-essential loads can be added to allow the generator to succor only important services (as hospital surgery for example).
\end{abstract}

\section{Methodology}

In the present paper, 8 innovative connections between diesel gensets are studied in order to allow the load to be fed by another neighboring genset in case of respective generator's failure.

The Reliability Block Diagram (RBD) model is applied to evaluate the reliability of every single electrical configuration here proposed [1-6].
Up to date reliability parameters [7-10] such as MTBF and failure rate $(\lambda)$, lead to accurate reliability calculations. By the hypothesis of constant failure rate of the components and components not repairable but quickly replaceable, the reliability calculation is performed and each system is compared in terms of MTBF [4].

\section{Failure rates}

In order to compare the reliability of each innovative solution by applying the RBD model, it is needed to use an up-to-date MTBF value of each single component used in each electrical configuration. The MTBF values are reported in Table 1.

Some of the proposed systems use a synchronization device, which failure's rate value was estimated. In terms of reliability, this device is mainly composed of an electronic control unit (ECU) and a circuit breaker (CB). Thus, by applying the RBD method it is found that its failure rate is the sum of the failure rates of these components. 
Table 1 Average MTBF of emergency generation system's components

\begin{tabular}{lcc}
\hline Components & Symbol & $\begin{array}{l}\text { Failure rate } \\
{[\text { failure/hr] }}\end{array}$ \\
\hline Automatic transfer switch & ATS & $9.795 \times 10^{-6}$ \\
Busbar & BB & $1.083 \times 10^{-6}$ \\
Contactor AC3/AC4 & CTT & $1 \times 10^{-8}$ \\
$\begin{array}{l}\text { Diesel genset } \\
\text { Electronic control unit }\end{array}$ & GE & $2 \times 10^{-5}$ \\
Load switch & ECU & $1.333 \times 10^{-6}$ \\
Synchronization device & LS & $4.160 \times 10^{-6}$ \\
$\begin{array}{l}\text { Thermal-magnetic circuit } \\
\text { breakers }\end{array}$ & SYNC & $5.681 \times 10^{-6}$ \\
\hline
\end{tabular}

$\lambda_{S Y N C}=\lambda_{E C U}+\lambda_{C B}$

It is highlighted that the contactor shows a noticeable higher reliability than an automatic circuit breaker's. Thus, the solutions with contactors instead of interrupters are preferred.

\section{Reliability Comparison between Different Emergency Systems Connections}

For sake of simplicity, only the best solutions are described with RBD schemes and calculations.

\subsection{Single standby generator (G1)}

In the single standby generator system, in case of utility failure (blackout), the load is fed by the standby generator after the commutation of an automatic-transfer switch, as shown in Fig. 1.

It can be evaluated that for the system G1, MTBF is equal to 3.8 years.

\subsection{Parallel configuration of standby generators (G2P, G3P)}

\subsubsection{Two standby generators in parallel (G2P)}

In the parallel configuration, when a blackout occurs, the load is fed by two standby generators connected in parallel with its respective synchronization devices, as shown in Fig. 2.

Applying the RBD method it can be found that, for the system G2P, the MTBF is equal to 4.1 years.

\subsubsection{Three standby generators in parallel (G3P)}

This configuration is similar to the previous one, with the difference that it is used an extra standby generator with its respective synchronization device. For simplicity, the system diagram is not shown; however it is composed by

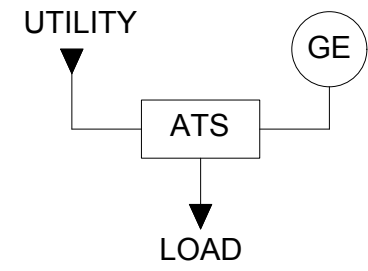

Fig. 1 Single standby generator (G1)

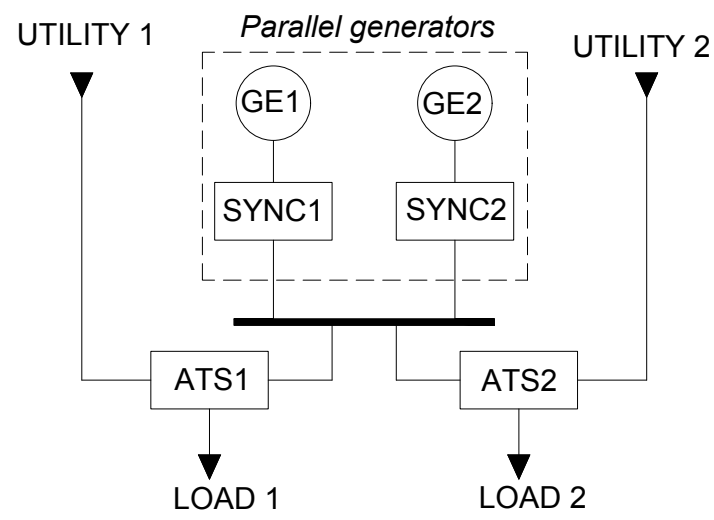

Fig. 2 Two standby generators in parallel (G2P)

three standby generators, each one connected through the synchronization device to the common bus bar (similar to Fig. 2). In this case, it is found that, for system G3P, MTBF is equal to 4.6 years.

\subsection{Two standby generators with succor bus tie between loads (G2S, G2C)}

In this configuration, when a blackout is detected, the electronic-control unit commands the opening of both breakers within the first automatic-transfer switch. Then, it commands the closing of the bus tie, located between loads, feeding one load from the power supply of the other one, as shown in Fig. 3.

Fig. 4 illustrates the reliability model studied for this configuration.

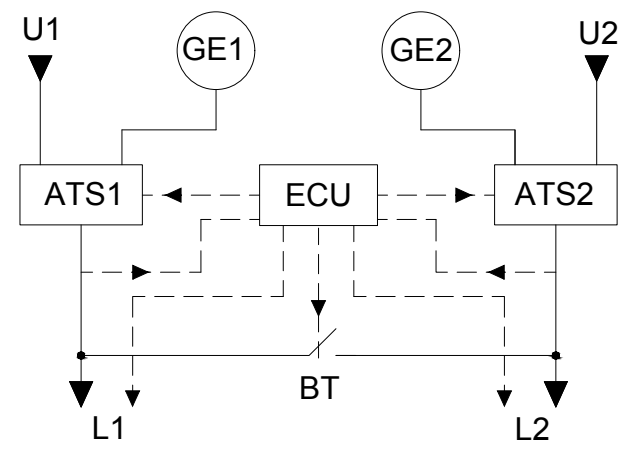

Fig. 3 Two standby generators with succor bus tie between loads (U: utility; L: load) 


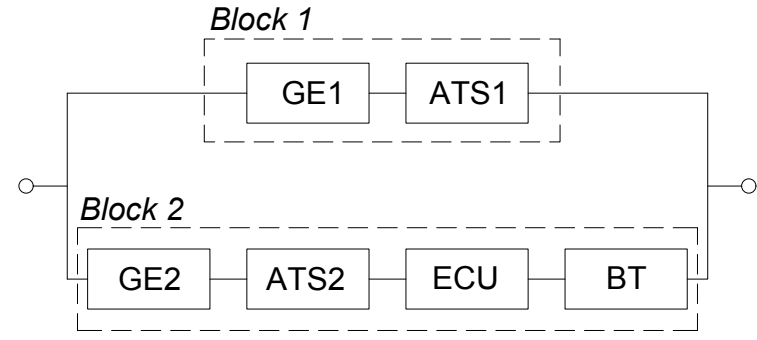

Fig. 4 RBD scheme of two standby generators with succor bus tie

Grouping the components in series and parallel blocks with their respective failure rate $(\lambda)$, the MTBF can be computed by means of the following equations, where $\lambda_{1}, \lambda_{2}$ are the failure rates of block 1 and 2 shown in Fig. 4, respectively; $R$ is the reliability function of the equivalent block.

$\lambda_{1}=\lambda_{G E 1}+\lambda_{A T S 1}$

$\lambda_{2}=\lambda_{G E 2}+\lambda_{A T S 2}+\lambda_{E C U}+\lambda_{B T}$

$R=R_{1}+R_{2}-R_{1} R_{2}$

$M T B F=\int_{0}^{\infty} R(t) d t$

\subsubsection{Two standby generators with succor load switch between loads (G2S)}

When a load switch is used as a bus tie it is found that the MTBF is equal to 5.3 years.

\subsubsection{Two standby generators with succor contactor between loads (G2C)}

In the case that a contactor it is used as a bus tie, (instead of the load switch), a MTBF value equal to 5.6 years is obtained.

\subsection{Two standby generators with mutual succor (G2MS, G2MC)}

\subsubsection{Two standby generators with mutual succor with load switch (G2MS)}

In this configuration, when both utility blackout and one standby generator starting failure are detected (through VGE and VU voltage detectors), the electronic control unit (ECU) commands the opening of both breakers (towards its own utility and local generator). Subsequently, the auxiliary ECU commands the start of the second standby generator and, finally, it commands the closure of the load switch (LS), feeding loads from the other standby generator, as shown in Fig. 5.

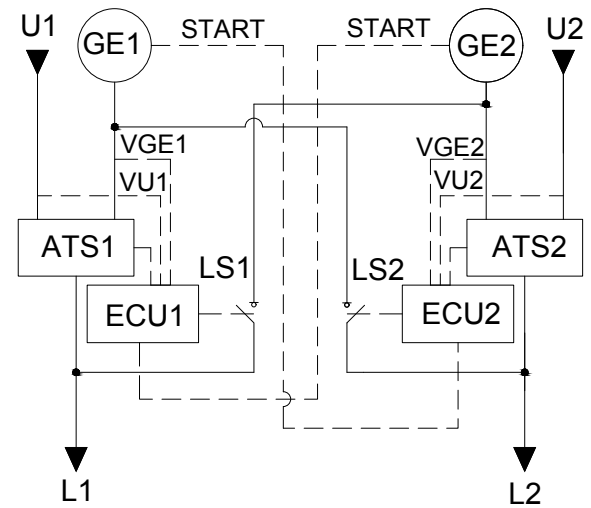

Fig. 5 Two standby generators with mutual succor with load switch (U: utility; L: load; VGE: Generator voltage detector; VU: Utility voltage detector)

For this configuration, it is found that the MTBF is equal to 6.2 years.

\subsubsection{Two standby generators with mutual succor with contactor (G2MC)}

This configuration is similar to the previous one, with the difference that, instead of a load switch, a contactor (CTT) is used to feed loads from the other standby generator, as shown in Fig. 6.

Fig. 7 illustrates the reliability model studied for this configuration.

Grouping the components in series and parallel blocks with their respectively failure rate $(\lambda)$, the MTBF can be computed by using the following equations, where $\lambda_{1}$, $\lambda_{2}$ are the failure rates of block 1 and 2 shown in Fig. 7, respectively; $R_{\mathrm{G} 2 \mathrm{MC}}$ is the reliability function of the equivalent block.

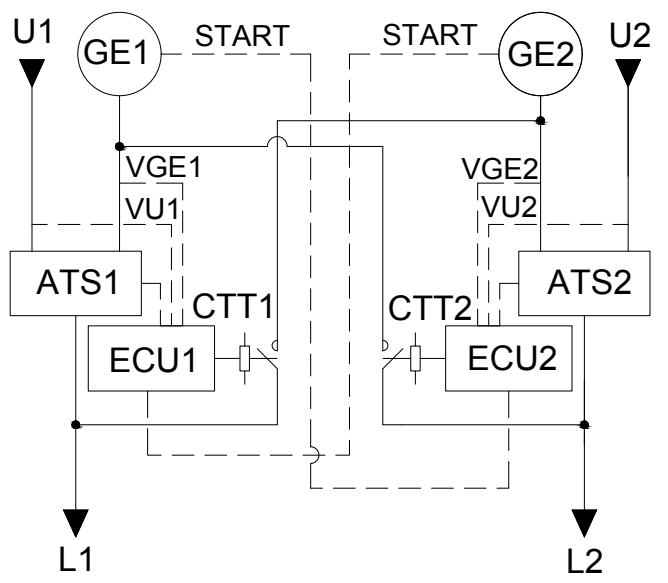

Fig. 6 Two standby generators with mutual succor with contactor (U: utility; L: load; VGE: Generator voltage detector; VU: Utility voltage detector) 


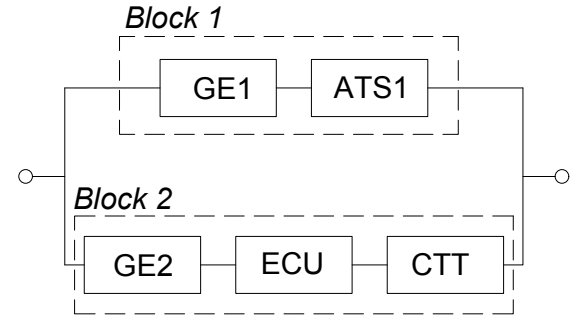

Fig. 7 RBD scheme of two standby generators with mutual succor with contactor

$\lambda_{1}=\lambda_{G E 1}+\lambda_{A T S 1}$

$\lambda_{2}=\lambda_{G E 2}+\lambda_{E C U}+\lambda_{C T T}$

$R_{G 2 M C}=R_{1}+R_{2}-R_{1} R_{2}$

$M T B F_{G 2 M C}=\int_{0}^{\infty} R_{G 2 M C} d t$

In this case, MTBF is equal to 7 years. It is highlighted the fact that the system's MTBF shows a noticeable improvement due to the high reliability of the contactor (as shown in Table 1).

\subsection{Three standby generators with mutual succor (G3MS, G3MC)}

In this configuration, every single load could be fed, in case of blackout, from its own standby generator or from the standby generator dedicated for the two adjacent loads, leading to a triangle configuration as shown in Fig. 8.

The electrical diagram for this configuration is shown in Fig. 9. For simplicity, it is only shown the case in which contactors are used to interconnect loads with the other standby generators.

\subsubsection{Three standby generators with mutual succor with load switch (G3MS)}

The electrical diagram for this configuration is similar to the one shown in Fig. 9, with the difference that a load switch (LS) is used instead of a contactor. The load switch is controlled by an electronic control unit (ECU) to perform the succor between the standby generators. In this case, MTBF is equal to 7.8 years.

\subsubsection{Three standby generators with mutual succor with contactor (G3MC)}

If a contactor (CTT) controlled by an electronic control unit (ECU) is used to perform the succor between the standby generators (as shown in Fig. 9), the MTBF is equal to 9 years.

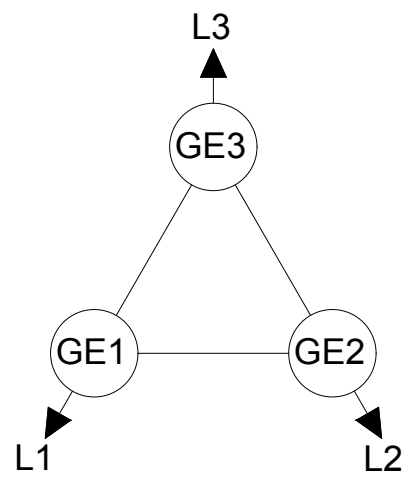

Fig. 8 Three standby generators with mutual succor configuration

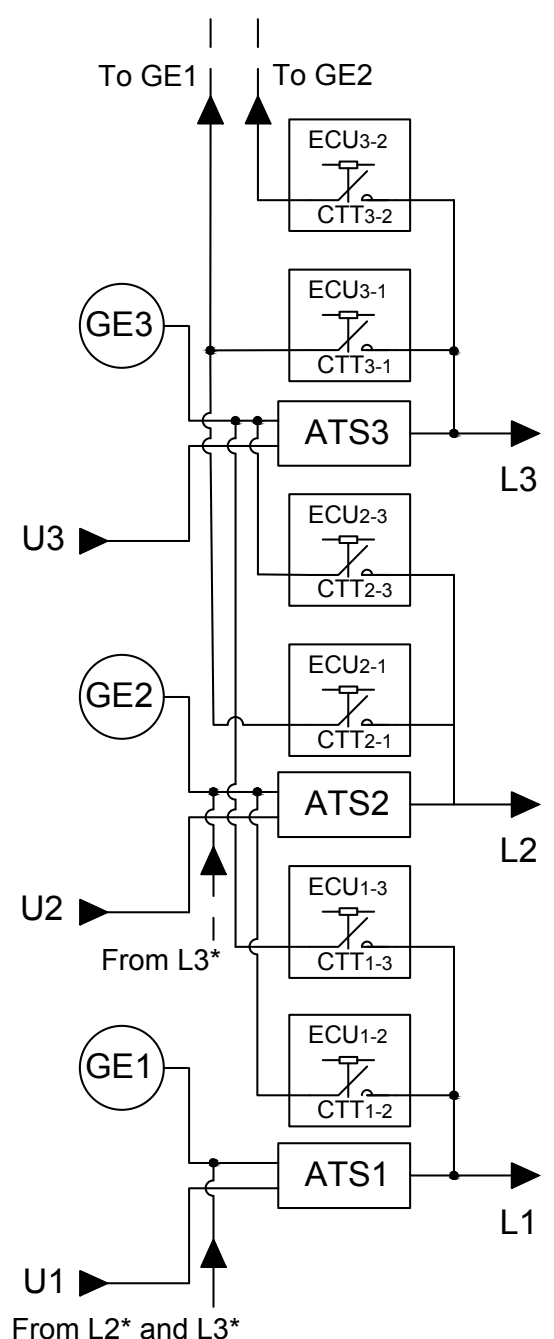

Fig. 9 Three standby generators with mutual succor with contactor (U: utility; L: load; L*: connection to the load through ECU and CTT block)

\section{Conclusions}

This paper proposed 8 different innovative solutions to improve the redundancy and reliability of standby generators systems. Fig. 10 illustrates the comparison between the MTBF values of each single studied configuration. 


\section{Comparison between configurations}

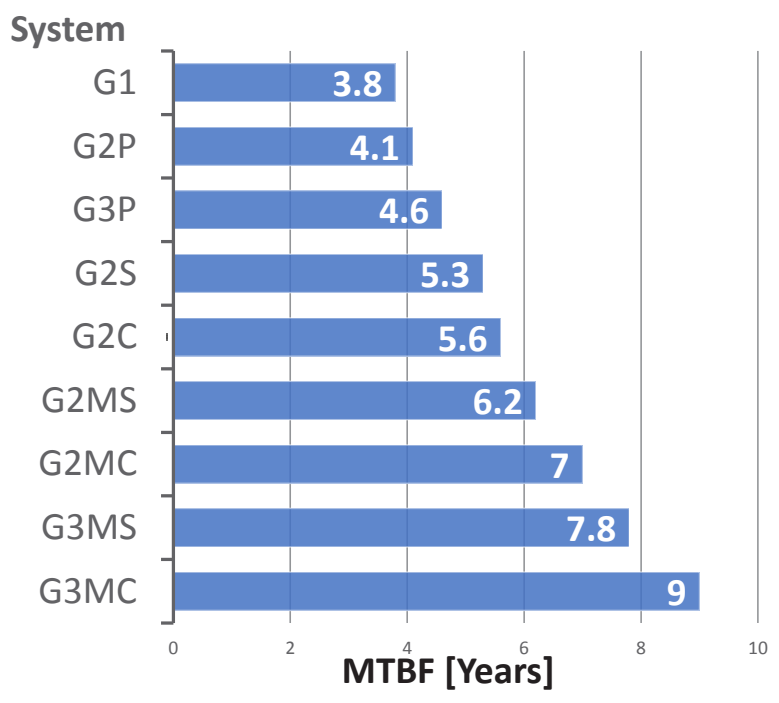

Fig. $10 \mathrm{MTBF}$ comparison between configurations

It is highlighted the practicality and technical feasibility of two main solutions. Considering not only the reliability but also technical and economic feasibility, the system "Two standby generators with mutual succor, with contactors $(G 2 M C)$ " is considered the best solution. It exhibits a MTBF of 7 years, improving of more than $80 \%$ the base solution (G1, which presents a MTBF of 3.8 years).

\section{References}

[1] Elia, S., Santantonio, A. "Fire Risk in MTBF Evaluation for UPS System", Advances in Electrical and Electronic Engineering, 14(2), pp. 189-195, 2016.

https://doi.org/10.15598/aeee.v14i2.1662

[2] Rausand, M., Hoyland, A. "System reliability theory: Models, Statistical Methods, and Applications", 2 ed., John Wiley \& Sons, USA, 2004.

[3] Rahmat, M. K., Jovanovic, S. "Reliability and Availability Estimation of DC Uninterruptible Power Supply Systems Using Monte-Carlo Simulation", In: 2015 IEEE 13th International Conference on Industrial Informatics (INDIN), Cambridge, UK, 2015, pp. 76-81.

https://doi.org/10.1109/INDIN.2015.7281713

[4] Khairil Rahmat, M., Jovanovic, S., Lo, K. L. "Uninterruptible Power Supply (UPS) System Configurations Reliability Comparison", In: 2010 IEEE International Conference on Power and Energy, Kuala Lumpur, Malaysia, 2010, pp. 835-840. https://doi.org/10.1109/PECON.2010.5697695

[5] Chiesa, S. "Affidabilita, sicurezza e manutenzione nel progetto dei sistemi", (Reliability, safety and maintenance for systems design), 1st ed., Clut, Turin, Italy, 2008. (in Italian)
The alternative solution, equally valid to the previous one, is the system "Two standby generators connected in the load side, by a contactor (G2C)". This system has a MTBF slightly lower (5.6 years) but it allows taking advantage also of the utility power supply of the other user. This calculation can be integrated with the MTBF of both utility (if connected to different supply) increasing the reliability of this configuration. Moreover this configuration is to be preferred because of its simplicity, low cost and easy maintenance.

It can be noticed that using standby generators in parallel does not improve so much the reliability of the system due to the presence of the synchronization devices.

The three standby generators systems show the best absolute reliability, however they are not recommended due to their high complexity and the difficulty of their maintenance and management; this complexity could cause the decrease of the MTBF due to human errors.

Furthermore, the use of two completely separate generation systems further improves reliability as it eliminates failures resulting from common mode causes. This configuration also facilitates maintenance operations.

\section{Acknowledgement}

This work was performed with the operative support on data collection by ENGIE maintenance supervisors Danilo Ebano and Mario Longo.

[6] Sulligoi, G., Vicenzutti, A., Tironi, E., Corti, M., Lamedica, R., Ruvio, A., Lipardi, G., Piva, L. "Naval smart grid: Integrated Power System for All Electric Naval Vessels with control and reliability characteristics", In: 2014 AEIT Annual Conference - From Research to Industry: The Need for a More Effective Technology Transfer (AEIT), Trieste, Italy, 2014, pp. 1-6. https://doi.org/10.1109/AEIT.2014.7002060

[7] Schneider Electric "Safe Machinery Handbook", Schneider Electric Inc., Rueil Malmaison, France, 2015.

[8] Institute of Electrical and Electronics Engineers, Inc. (IEEE) "STD 493-2007 Recommended Practice for the Design of Reliable Industrial and Commercial Power Systems", IEEE, USA, 2007. https://doi.org/10.1109/IEEESTD.2007.380668

[9] MIL-HDBK-217F "Military handbook reliability prediction of electronic equipment", US Department of Defense, USA, 1991.

[10] Rahmat, M. K., Jovanovic, S., Lo, K. L. "Reliability Modelling of Uninterruptible Power Supply Using Probability Tree Method", In: Proceedings of the 41st International Universities Power Engineering Conference, Newcastle-upon-Tyne, UK, 2006, pp. 603-607. https://doi.org/10.1109/UPEC.2006.367549 